\title{
Diagnóstico del compromiso ambiental de las instituciones de educación superior y técnica costarricenses ${ }^{*}$
}

\section{Assessment of the Environmental Commitment of Costa Rican Higher and Technical Education Institutions}

\author{
José Francisco Calderón Zúñiga** \\ Manrique Arguedas Camacho**
}

DOI: 10.22458/rb.v32i2.3877

Recibido -Received: 30/06/2021 / Corregido - Revised: 27/07/2021 / Aceptado - Accepted: 07/10/2021

\section{RESUMEN}

El propósito del trabajo es determinar el estado de la situación del compromiso ambiental de las instituciones educativas superior y técnicas (IEST) de Costa Rica, utilizando una encuesta ya aplicada en otros países, la cual se origina en otro estudio más extenso ya realizado. Se busca entender la dimensión del verdadero quehacer de las instituciones mediante la aplicación de la encuesta de 25 preguntas cerradas y dos preguntas abiertas complementarias. Cada una de las preguntas suma un punto en la evaluación y a más puntos mejor calificación. Ciertamente, los resultados a nivel general de la muestra obtenida colocan al país en un nivel medio de institucionalización del compromiso ambiental con oportunidades de mejora en los ámbitos de docencia $(2,6)$ e investigación $(1,8)$ y sobresale los ámbitos de gobierno $(3,4)$ y gestión $(3,4)$ y las IEST miembros de la Red Costarricense de Instituciones Educativas Sostenibles (REDIES) aventajan a las que no forman parte de esta Red, según los resultados obtenidos.

Palabras clave: concientización; compromiso; REDIES; sostenibilidad.

\section{ABSTRACT}

The purpose of this investigation is to determine the status of the environmental commitment situation of the Higher and Technical Educational Institutions (IEST by its acronym in Spanish) of Costa Rica using a survey already applied in other countries, which originates from another more extensive, already carried out, study. This instrument seeks to understand the scope of real work by the institutions through the application of a survey of 25 closed questions and two more complementary open questions, with each of the questions adding one point in the evaluation, where the greater the number of points, the better the score. Certainly, the general sample results obtained place the country at an intermediate level of institutionalization of environmental commitment with opportunities for improvement in the fields of teaching (2.6), research (1.8), the government $(3,4)$, and management $(3,4)$. In addition, IEST members of the Costa Rican Network of Sustainable Educational Institutions (REDIES) were revealed to outperform those that are not part of this Network according to the results obtained.

Keywords: awareness; commitment; REDIES; sustainability.

* Artículo de investigación. Iniciativa de La Red Costarricense de Instituciones Educativas Sostenibles, coordinado por la Unidad de Acción Ambiental de la Universidad EARTH.

* Estudiante de la carrera de Bachillerato en Ingeniería Industrial de la Universidad Autónoma de Centro América.jfcaldez@hotmail.es ID: https://orcid.org/0000-0001-8664-9393

** Coordinador de la Unidad de Acción Ambiental de la Universidad EARTH y Docente de la Universidad de Costa Rica. Máster en Mercadeo y Administración de Negocios. margueda@earth.ac.cr

ID: https://orcid.org/0000-0001-8559-9099 


\section{Introducción}

La Red Costarricense de Instituciones Educativas Sostenibles (REDIES) busca lograr el compromiso de las autoridades en las instituciones educativas participantes para alcanzar la sostenibilidad en los campus, por medio de indicadores de sostenibilidad, y se dirige tanto a universidades públicas como privadas del país. Además, se promueve el establecimiento de alianzas estratégicas en el tema de sostenibilidad que favorezcan el intercambio y la cooperación técnica, mediante la ejecución de acciones sostenibles entre las instituciones miembros y las comunidades que la conforman.

Para lograr lo anterior y como parte de sus objetivos, REDIES ha desarrollado una serie de indicadores que reflejan en su mayoría el quehacer de las instituciones miembros en su gestión ambiental. Por tanto, se elabora -mediante una encuesta- el diagnóstico sobre la inclusión de consideraciones ambientales en las instituciones, tomando como base el cuestionario realizado por la Red de Indicadores de Sostenibilidad en Universidades (RISU) aplicado en diez países de Latinoamérica y 328 universidades, el estudio original abarcó 114 indicadores distribuidos en 11 temáticas o dimensiones de la posible aplicación de la sostenibilidad en universidades (RISU, 2014).

REDIES ha promovido la continuidad del estudio aplicándolo en las instituciones en educación superior y técnica de Costa Rica. El mismo instrumento ha sido aplicado en otros países miembros de la Alianza Iberoamericana de Universidades por la Sustentabilidad y el Ambiente (ARIUSA).

\section{Antecedentes de la investigación}

Como parte de una preocupación general de un grupo de instituciones en educación superior y técnica respecto al quehacer en términos de sostenibilidad y ambiente, en el año 2009 surge la iniciativa liderada por la Universidad EARTH para el "Primer Encuentro Interinstitucional: Perspectivas para la sostenibilidad en organizaciones de educación superior costarricenses".

Del primer acercamiento surge la formación de REDIES en el año 2010, lo cual propició el intercambio de experiencias, conocimiento y acciones conjuntas entre las instituciones que la conforman. Desde sus inicios REDIES ha venido trabajando en diferentes aspectos, estrategias y alianzas en pro de incorporar el eje ambiental como parte de la gestión propia de las IEST, aportando y promoviendo la aplicación, propuesta y diseño de las diferentes estrategias que propicien el desarrollo de un entorno más amigable y sostenible.

\section{Materiales y métodos}

El estudio es de tipo cuantitativo con un alcance exploratorio. Los estudios exploratorios se realizan cuando el objetivo es examinar un tema o problema de investigación poco estudiado, del cual se tienen muchas dudas o no se ha abordado (Sampieri, 2010). De esta manera, la investigación nos permitirá familiarizarnos con situaciones desconocidas dando origen a identificar conceptos, problemas o variables que formulen nuevas investigaciones, pues todo conocimiento es aproximado y relativo, de tal modo que nunca se considera acabado (Rivero, 2008). 
Herramientas de recolección

y ámbitos de evaluación

Para la captura de la información se utilizó la plataforma de Google Forms para establecer una encuesta en línea, utilizando las 25 preguntas definidas por RISU y dos preguntas complementarias, la misma fue enviada a las instituciones educativas (67 instituciones en total) dirigida a los representantes o las personas clave de la institución que cuentan con la información suficiente para poder responder con propiedad.

\section{Características de la encuesta}

El instrumento para la captura de información fue diseñado de manera tal que al aplicarlo fuese posible comparar los resultados del país (Costa Rica) con los resultados de los otros países que han desarrollado la encuesta. Sin embargo, el alcance del estudio no implica realizar la comparación y se consideraron solo las instituciones de educación superior y técnica en Costa Rica.
El diseño del instrumento aplicado incluye preguntas dicotómicas con respuestas de sí y no en su mayoría, proporcional a cada ámbito de acción, como se aprecia en la tabla 1. El sujeto fuente de información fueron personas que manejan mayormente los temas ambientales o los responsables de temas relacionados en sus instituciones. El instrumento fue aplicado mediante la plataforma de Google Forms, del 10 de septiembre al 19 de octubre del año 2018.

Fuera de las 25 preguntas originales, se adicionaron dos preguntas complementarias abiertas de libre respuesta. El número 26 "Si respondió positivamente en la pregunta 5: Indique los mecanismos o espacios institucionales que faciliten la participación de los estudiantes en la gestión ambiental de la universidad" y el 27 "Si respondió positivamente en la pregunta 7: Indique el programa académico de la universidad que se considera más avanzado en la incorporación de la perspectiva de la sustentabilidad ambiental". Las preguntas ayudarán más adelantes a comprender la información aportada por cada institución.

Tabla 1

Encuesta para el diagnóstico de la Institucionalización del compromiso ambiental de las universidades colombianas

\begin{tabular}{|c|c|}
\hline $\begin{array}{l}\text { Ámbito de } \\
\text { acción }\end{array}$ & Preguntas básicas \\
\hline \multirow{5}{*}{$\begin{array}{l}\text { GOBIERNOY } \\
\text { PARTICIPACIÓN } \\
\text { AMBIENTAL }\end{array}$} & $\begin{array}{l}\text { 1. ¿Existe un documento que defina la política que la universidad desarrolla en el ámbito de la sustentabilidad o } \\
\text { el ambiente? }\end{array}$ \\
\hline & $\begin{array}{l}\text { 2. ¿Existe una unidad, oficina o servicio de carácter técnico-administrativo con dedicación exclusiva para los } \\
\text { temas de sustentabilidad ambiental? }\end{array}$ \\
\hline & $\begin{array}{l}\text { 3. La organización de la acción por la sustentabilidad ambiental en su universidad corresponde a: a) un } \\
\text { conjunto de acciones o agenda; b) un plan de acción ambiental; c) un sistema ambiental institucional }\end{array}$ \\
\hline & $\begin{array}{l}\text { 4. ¿La unidad, oficina o servicio de sustentabilidad ambiental dispone de presupuesto propio para realizar } \\
\text { actividades o inversiones en esta materia? }\end{array}$ \\
\hline & $\begin{array}{l}\text { 5. ¿Están involucrados los docentes, estudiantes o administrativos en el diseño e implementación de la política } \\
\text { ambiental o de sustentabilidad en la universidad? }\end{array}$ \\
\hline
\end{tabular}


6. ¿Existe en la política ambiental o de sustentabilidad de la universidad mención expresa a las actividades docentes o de formación?

7. ¿Se incorpora en algunos de los currículos académicos de las distintas carreras la perspectiva de la sustentabilidad ambiental?

DOCENCIA Y

FORMACIÓN

AMBIENTAL

8. ¿Se han definido de manera explícita competencias o capacidades transversales básicas en sustentabilidad ambiental en algunas carreras que ofrece la universidad?

9. ¿Existen en la universidad programas de grado o pregrado (carreras profesionales) específicos sobre ambiente o sustentabilidad?

10. ¿Existen en la universidad programas de posgrado (especializaciones, maestrías o doctorados) específicos sobre ambiente o sustentabilidad?

11. ¿Existe en la política ambiental o de sustentabilidad de la universidad mención expresa a la investigación y transferencia de tecnología?

12. ¿Existe en la universidad algún grupo de investigación específico sobre ambiente o sustentabilidad formalmente constituido?

INVESTIGACIÓN

Y TECNOLOGÍA

AMBIENTAL

13. ¿La universidad ha participado en proyectos nacionales o internacionales de investigación sobre ambiente o sustentabilidad?

14. ¿Se consideran criterios de sustentabilidad y evaluación del impacto ambiental en la aprobación, por parte de la universidad, de los proyectos de investigación y transferencia de tecnología?

15. ¿Existen estrategias para incentivar la utilización de la universidad y de sus campus o instalaciones en la realización de investigaciones sobre temas ambientales y de sustentabilidad?

16. ¿Se implementa un plan específico, eje estratégico o plan de acción de responsabilidad socioambiental que ha sido aprobado por algún órgano de gobierno de la universidad?

17. ¿Existen programas permanentes de intervención para contribuir a la solución de problemas ambientales 0 de sustentabilidad en comunidades?

EXTENSIÓNO

PROYECCIÓN

AMBIENTAL

GESTIÓNY

ORDENAMIENTO

AMBIENTAL
18. ¿Existen programas permanentes de colaboración con gobiernos (locales, regionales o nacionales) para la formulación o implementación de políticas públicas ambientales o de sustentabilidad?

19. ¿Existen programas permanentes de vinculación con el sector empresarial del entorno en materia de ambiente o sustentabilidad?

20. ¿Participa la universidad en redes universitarias nacionales o internacionales por el ambiente o la sustentabilidad?

21. ¿Se implementa un plan específico, eje estratégico o línea de acción de sustentabilidad ambiental que incluya aspectos de ahorro en agua, sanidad para consumo humano, riego y gestión de aguas residuales?

22. ¿Se implementa un plan específico, eje estratégico o línea de acción de sustentabilidad ambiental que incluya aspectos relacionados con alumbrado, climatización y energías renovables?

23. ¿Se implementa un plan específico, eje estratégico o línea de acción de sustentabilidad ambiental para la minimización, recogida selectiva y manejo adecuado de los residuos sólidos?

24. ¿Se implementa un plan específico, eje estratégico o línea de acción de sustentabilidad o ambiental sobre la gestión de las zonas verdes y la biodiversidad?

25. ¿La planificación urbanística de la universidad incluye criterios de sustentabilidad o ambientales?

Nota. Tomado de informe Colombia "Institucionalización del compromiso ambiental de las universidades colombianas". 
Escalas de medición

Cada ámbito de acción se evalúa con una escala específica y una escala general dada por la suma de las respuestas positivas de cada institución, ambas utilizadas en otros estudios de la región.

Cada pregunta afirmativa sumó un punto, por lo que el máximo de puntos obtenidos fue de 25, cada ámbito de acción fue agrupado en cinco preguntas; según la suma de preguntas positivas así se ubicó el nivel de compromiso en la escala de evaluación. La escala por nivel de compromiso obtenido se observa en la tabla 2.

Tabla 2

Niveles de compromiso ambiental de los ámbitos de acción

\begin{tabular}{cc}
\hline Niveles & Puntajes \\
\hline Muy Bajo & $0,0-1,0$ \\
\hline Bajo & $1,1-2,0$ \\
\hline Medio & $2,1-3,0$ \\
\hline Alto & $3,1-4,0$ \\
\hline Muy Alto & $4,1-5,0$ \\
\hline
\end{tabular}

Nota. Tomado de informe Colombia "Institucionalización del compromiso ambiental de las universidades colombianas".

El desempeño general, al igual que por ámbito de acción, se calificó según la escala general, la cual acumula y define diferentes rangos de compromiso institucional, cada rango equivale a 5 puntos que van desde un nivel muy bajo con un máximo de 5 puntos hasta un nivel muy alto con 25 puntos, tal como se puede observar en la tabla 3.
Tabla 3

Escala general para medir el nivel de compromiso de las instituciones de educación

\section{Niveles}

Puntajes

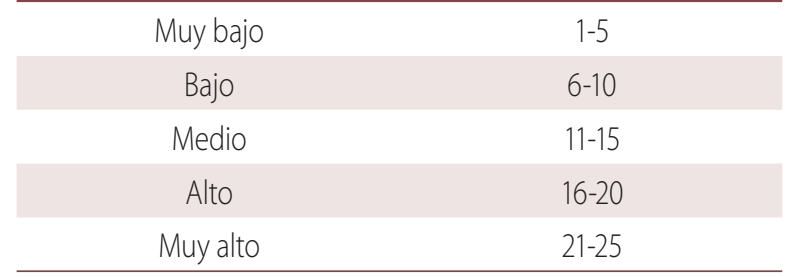

Nota. Tomado de informe Colombia "Institucionalización del compromiso ambiental de las universidades colombianas".

\section{Población y muestra}

La invitación para participar del estudio se envió a 67 IEST, de las cuales 24 atendieron en forma oportuna y enviaron los resultados. Respondieron la encuesta $100 \%$ de las universidades públicas del país y hubo una participación significativa de universidades privadas, la muestra obtenida es de $36 \%$ del total de las IEST en el país.

Para el año 2015, en Costa Rica existía una matrícula de 208008 estudiantes en educación superior (Programa Estado de la Nación, 2017), para esa misma fecha el total de estudiantes de las universidades adheridas a REDIES era de 76 012, lo que representa 37\% de la población estudiantil en educación superior y años más tarde la cifra crecería significativamente. La distribución de las instituciones consultadas, cuyas sedes principales se encuentran distribuidas a lo largo del país se puede observar en la tabla 4.

A partir de la información anterior, se observa que la concentración mayor de las sedes principales geográficamente se ubica en San José; no obstante, muchas de las instituciones cuentan con sedes en las otras seis provincias. 
Tabla 4

Distribución de participación por provincia

\section{Ciudad de la sede principal}

de la institución

\begin{tabular}{lcc} 
San José & 18 & $75 \%$ \\
\hline Alajuela & 2 & $8 \%$ \\
\hline Cartago & 2 & $8 \%$ \\
Limón & 1 & $4 \%$ \\
Heredia & 1 & $4 \%$ \\
\hline Total & 24 & $100 \%$ \\
\hline
\end{tabular}

Nota. Elaboración propia con base en la muestra obtenida.

\section{Resultados y discusión}

Para el estudio se envió la invitación a 67 instituciones de educación, de las cuales 21 (31\%) pertenecen a la Red Costarricense de Instituciones de Educativas Sostenibles (REDIES) y el restante $69 \%$ a otros centros de estudio. Se obtiene una participación de 24 instituciones que representan $35 \%$ del total, cuatro de las IEST no pertenecen a REDIES; sin embargo, analizando la participación en términos de población estudiantil y por casa de estudio, se evidencia mayor peso en el impacto general de las instituciones participantes al considerar al alumnado como población de la muestra.

En 2015, el quinto año en que se dispuso de la información sobre la matrícula total de las universidades costarricenses, el Consejo Nacional de Rectores (CONARE) reportó la cifra de 208008 estudiantes inscritos con lo que la cobertura de la población en edad de asistir a la educación superior alcanza $48,5 \%$, si se considera el grupo de 18 a 22 años (Estado de la Educación, 2017), y según la información recopilada desde REDIES sus integrantes reportaron 245550 estudiantes para 22 instituciones que la integraban en el 2016.

Uno de los más importantes resultados corresponde al nivel de institucionalización del compromiso ambiental, para el estudio se obtiene una media de 13,9 puntos que ubica en el nivel medio del compromiso ambiental; sin embargo si observamos las IEST que se encuentran de medio hacia muy alto, corresponde $63 \%$ o lo que es igual a 15 instituciones que están en niveles aceptables, considerando que la mayoría son miembros de REDIES y trabajan en los aspectos consultados, $17 \%$ se ubican en un nivel muy bajo, $21 \%$ en el segmento bajo, $17 \%$ en un nivel medio, $25 \%$ alto y $21 \%$ en la escala de muy alto. En la tabla 5 se puede apreciar la clasificación completa.

Tabla 5

Nivel de institucionalidad del compromiso ambiental

\begin{tabular}{cccc}
\hline Grupo & Frecuencia & \% Relativo & \% Acumulado \\
\hline Muy bajo & 4 & $17 \%$ & $17 \%$ \\
\hline Bajo & 5 & $21 \%$ & $38 \%$ \\
\hline Medio & 4 & $17 \%$ & $54 \%$ \\
\hline Alto & 6 & $25 \%$ & $79 \%$ \\
\hline Muy alto & 5 & $21 \%$ & $100 \%$ \\
\hline
\end{tabular}

Nota. Elaboración propia con base en los resultados.

Si bien es cierto, los resultados anteriores muestran una importante segmentación de las organizaciones del nivel medio hacia arriba de la tabla, un porcentaje considerable (38\%) está en los niveles bajo y muy bajo. La mayor concentración se encuentra en un nivel alto con seis instituciones. 
Tabla 6

Datos obtenidos sobre institucionalidad del compromiso ambiental

\begin{tabular}{cc}
\hline Media & 13,9 \\
\hline Moda & 5,0 \\
\hline Mediana & 14,0 \\
\hline Desviación & 7,0 \\
\hline Máximo & 25,0 \\
\hline Mínimo & 1,0 \\
\hline
\end{tabular}

Nota. Elaboración propia con base en los resultados.

La información aportada por la encuesta en la tabla 6 revela que el grupo de universidades tiene una media de 13,9 respuestas positivas, lo que las ubica en un nivel medio de institucionalización del compromiso ambiental, la media es de 14 e indica que la mitad de las instituciones que respondieron están por encima de un nivel medio y que la desviación estándar es de 7, además de que solo una institución logra el puntaje máximo de 25 y una el menor puntaje con solo una respuesta positiva, como lo ilustra el gráfico 1.

En el gráfico 1 se observa que nueve instituciones se encuentran entre los niveles bajo y muy bajo, tres coinciden con 5 puntos y otras tres coinciden con 8 puntos de 25 . De las nueve, cinco pertenecen a REDIES y corresponde a $28 \%$ de los integrantes de la red, el restante $72 \%$ de los miembros de la red se distribuyen en $11 \%$ (2) en el nivel medio, $33 \%$ (6) en el nivel alto y $28 \%$ (5) en la posición muy alto.

\section{Ámbitos de acción}

Al visualizar los resultados agrupados por ámbitos de acción, como se aprecia en la tabla 7, es posible identificar el desempeño general y la clasificación de las IEST, mismos resultados que más adelante serán analizados de forma separada entre los miembros y no miembros de REDIES como lo ilustra el gráfico 2.

En la tabla 7, se puede apreciar que los ámbitos de gobierno y gestión obtienen las puntuaciones más altas con 3,4 puntos de una escala máxima de 5 , seguidos por extensión $(2,7)$ y docencia $(2,6)$ y con el puntaje más bajo investigación $(1,8)$.

\section{Gráfico 1}

Puntaje general del compromiso ambiental de las 24 instituciones de educación costarricenses

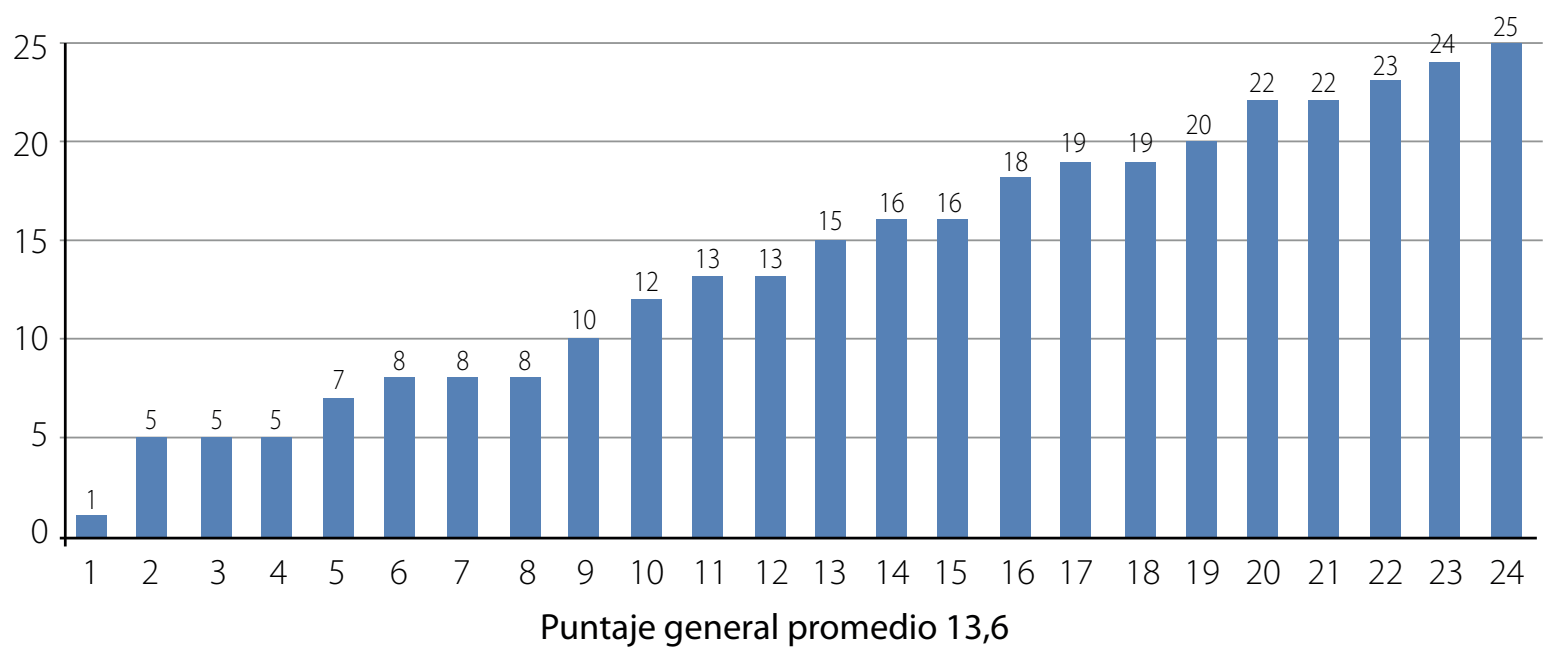


Tabla 7

Resultados por ámbitos de acción

\begin{tabular}{|c|c|c|c|c|}
\hline \# & $\begin{array}{l}\text { Ámbito de } \\
\text { acción }\end{array}$ & $\begin{array}{c}\mathrm{N}^{0} \mathrm{de} \\
\text { preguntas }\end{array}$ & General & Clasificación \\
\hline 1 & GOBIERNO & 1 a 5 & 3,4 & Alto \\
\hline 2 & $\begin{array}{l}\text { DOCENCIA } \\
\text { YFORMACIÓN }\end{array}$ & 6 a 10 & 2,6 & Medio \\
\hline 3 & $\begin{array}{l}\text { INVESTIGACIÓN } \\
\text { YTECNOLOGÍA }\end{array}$ & 11 a 15 & 1,8 & Bajo \\
\hline 4 & $\begin{array}{l}\text { EXTENSIÓN } \\
\text { OPROYECCIÓN }\end{array}$ & 16 a 20 & 2,7 & Medio \\
\hline 5 & $\begin{array}{l}\text { GESTIÓN } \\
\text { YORDENAMIENTO }\end{array}$ & 21 a 25 & 3,4 & Alto \\
\hline & Promedio & 2,8 & & Medio \\
\hline
\end{tabular}

Nota. Elaboración propia con base en los resultados.

Al clasificarlos, según la tabla 2, por nivel de compromiso ambiental, se ubican en un nivel alto dos de los cinco ámbitos, gobierno y gestión; en un nivel medio los ámbitos de docencia y extensión y por último en un nivel bajo el ámbito de investigación. Es de notar que no se logra el nivel muy alto que estaba entre el rango de 4,1 a 5 .

Se aprecia un promedio de 2,8 que ubica el desempeño general de las IEST en un nivel medio del compromiso ambiental, según la escala.

\section{Gráfico 2}

Resultados por ámbitos de acción en instituciones miembros y no miembros de REDIES

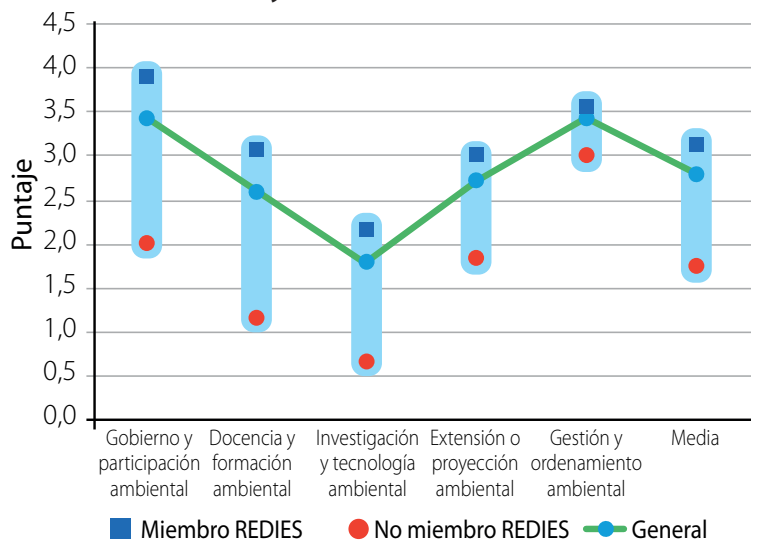

Nota. Elaboración propia con base en los resultados.
En el gráfico 2 están agrupados los resultados por IEST miembros de REDIES, no miembros y los resultados generales.

Resulta evidente la diferencia entre los miembros y no miembros de REDIES, en los ámbitos de gobierno y docencia la brecha es de 1,9 puntos los más amplios y en gestión la menor diferencia de 0,6 puntos. Con respecto al ámbito de investigación, es el que presenta mayores oportunidades de mejora para ambos grupos y un desafío importante para las IEST costarricenses.

\section{Preguntas de evaluación}

La encuesta está diseñada de manera que permite medir aspectos más específicos del compromiso por parte de las instituciones. En el gráfico 3 se presentan los resultados afirmativos por número de pregunta. Los resultados individuales brindan mayor detalle sobre el estado actual del nivel de institucionalización del compromiso ambiental, así como el identificar los aspectos puntuales de cada una de las preguntas.

La pregunta 3, al referirse a la organización de la acción por la sustentabilidad, no se responde como sí o no, por lo que en el gráfico 3 no tiene valores asociados; no obstante, suma un punto a todas las IEST cuando se valoran el total de repuestas positivas por institución.

De las preguntas, $58 \%$ fueron respondidas de manera positiva y el rango de respuestas positivas va de 6 a 22, en el rango mínimo con 6 respuestas positivas solo se encuentra la pregunta 14 y solo una pregunta en el máximo de 22 (la pregunta 20). 


\section{Gráfico 3}

Número de respuestas positivas por pregunta

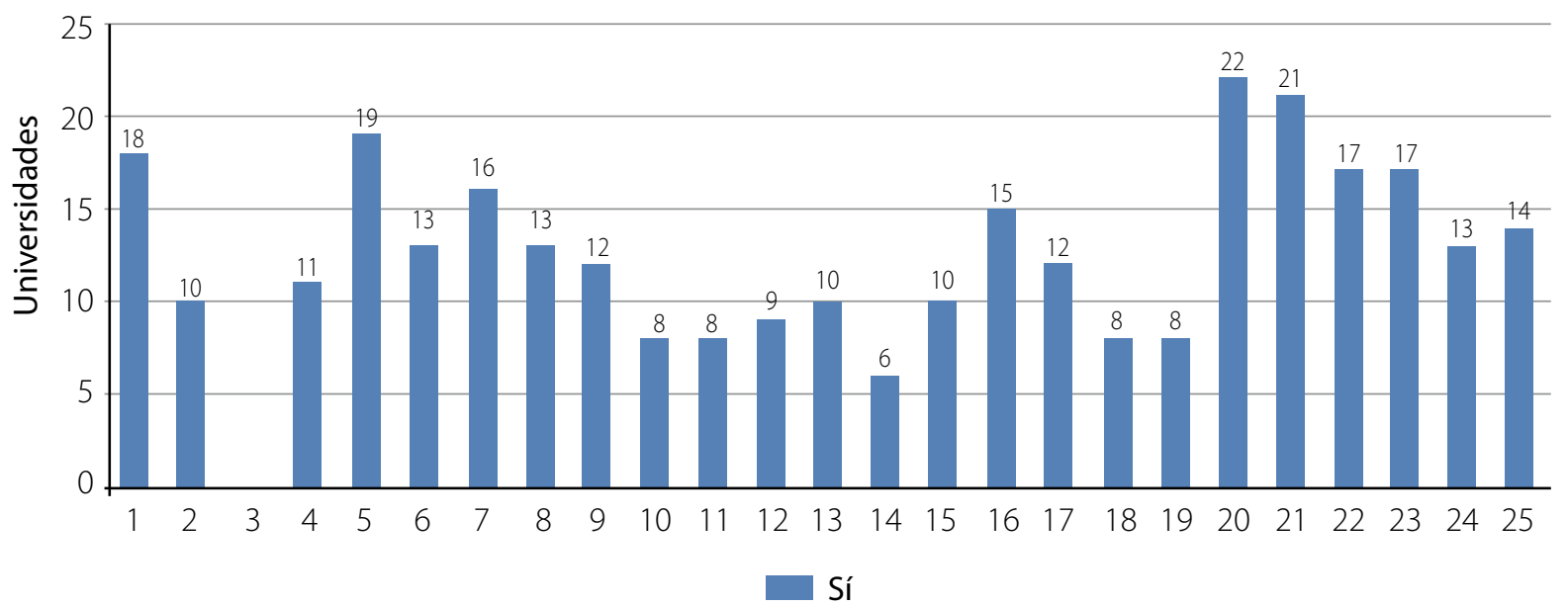

Nota. Elaboración propia con base en los resultados.

Al observar el gráfico 3, destaca el hecho de que las cinco preguntas con mayor cantidad de respuestas positivas son la 20,21, 5, 1 y 22 , respectivamente. Preguntas relacionadas con la existencia de la política ambiental, los programas de gestión de energía y aguas, además de si participan las IEST en redes, que en este caso es la de mayor puntuación; aspecto esperado ya que $75 \%$ de las IEST que respondieron son miembros de REDIES.

Al otro extremo, hallamos el grupo de preguntas con menor número de respuestas positivas la 10,11, 18, 19 y 14, del grupo ninguna superó las 8 respuestas positivas y la de menor puntaje con solo 6 respuestas positivas fue la 14 , relativa a la incorporación de criterios de sustentabilidad y evaluación de impacto ambiental en la aprobación de proyectos por parte de las IEST.

Como se mencionó anteriormente, una de las preguntas que no se responde como sí o no es la tercera, la pregunta indaga sobre la organización que utiliza las IEST en su accionar por la sustentabilidad ambiental y las posibles respuestas ofrecidas fueron: a) un conjunto de acciones o agenda; b) un plan de acción ambiental; c) un sistema ambiental institucional. Los resultados se pueden observar en la tabla 9.

Tabla 8

Resultados de la pregunta 3

\begin{tabular}{lcc}
\multicolumn{1}{c}{ Respuesta } & Frecuencia & $\%$ \\
\hline Un conjunto de acciones o agenda & 8 & $33 \%$ \\
Un Plan de Acción Ambiental & 13 & $54 \%$ \\
\hline Un Sistema Ambiental Institucional & 3 & $13 \%$ \\
\hline Nota. Elaboración propia con base en los resultados.
\end{tabular}

Según la tabla 8, 54\% (13) de las IEST indica que su organización es afín a un plan de acción ambiental y 33\% (8) de las instituciones lo indican como un conjunto de acciones o agenda, mientras $13 \%$ (3) como un sistema ambiental institucional.

Segregando los resultados de las IEST, según su propia clasificación de la pregunta 3 en la tabla 9, es posible identificar que aquellas que trabajan los aspectos ambientales mediante el establecimiento de un plan de 
acción ambiental resultaron con puntuaciones que las colocan en niveles arriba de medio en la clasificación del compromiso ambiental, en contraste las que obtienen niveles altos y muy altos.

Lo anterior nos brinda una perspectiva que posiciona el modelo de sistema ambiental institucional como un medio efectivo. Aunque no es el alcance del estudio analizarlo con detalle, definitivamente es una tarea por realizar en futuros trabajos.

Tabla 9

Clasificación de resultados de la pregunta 3

\begin{tabular}{lcc|}
\hline \multicolumn{1}{c}{ Respuesta } & Frecuencia & $\%$ \\
\hline Un conjunto de acciones & 8 & $100 \%$ \\
o agenda & 1 & $13 \%$ \\
\hline Alto & 4 & $50 \%$ \\
\hline Bajo & 2 & $25 \%$ \\
\hline Medio & 1 & $13 \%$ \\
\hline Muy alto & 13 & $100 \%$ \\
\hline Un plan de acción ambiental & 4 & $31 \%$ \\
\hline Alto & 1 & $8 \%$ \\
\hline Bajo & 2 & $15 \%$ \\
\hline Medio & 2 & $15 \%$ \\
\hline Muy alto & 4 & $31 \%$ \\
\hline Muy bajo & 3 & $100 \%$ \\
\hline Un sistema ambiental & & $33 \%$ \\
\hline institucional & 1 & $67 \%$ \\
\hline Alto & 2 & \\
\hline Muy alto & & \\
\hline
\end{tabular}

Nota. Elaboración propia con base en los resultados.

Observando el porcentaje de respuestas positivas según los ámbitos de acción, es notorio que en cuatro de los cinco ámbitos los porcentajes de respuestas positivas superan 50\%, a excepción del ámbito de investigación y tecnología ambiental.
En la tabla 10, al segregar los resultados se evidencia que el promedio de respuestas positivas para las IEST no adscritas a la red es de 8,67 ubicándolas en un nivel bajo de compromiso ambiental y las IEST miembros con un promedio de 15,67 colocándolas en un nivel alto de compromiso ambiental.

Tabla 10

Respuestas positivas IEST miembros y no miembros de REDIES

$\begin{array}{ccc}\text { Miembro REDIES } & \text { Promedio } & \text { Nivel } \\ \text { No } & 8,67 & \text { Bajo } \\ \text { Sí } & 15,67 & \text { Alto } \\ \text { General } & 13,9 & \text { Medio }\end{array}$

Nota. Elaboración propia con base en los resultados.

Los resultados obtenidos resaltan como una de las mayores debilidades el ámbito de investigación y tecnología ambiental con un nivel bajo, seguido por docencia y formación ambiental con un nivel medio.

La principal fortaleza de las IEST costarricenses se destaca en el ámbito de gestión y ordenamiento ambiental donde $68 \%$ responde de manera positiva. Como elemento adicional, se incluyeron dos preguntas complementarias abiertas para ampliar la interpretación de dos preguntas, la pregunta 26 asociada a la pregunta 5 y la pregunta 27 asociada a la pregunta 7 , respectivamente.

Para la pregunta 26 , se contabilizó $66,6 \%$ (16) de las 24 IEST que ampliaron con uno o más mecanismos que facilitan la participación de los estudiantes en la gestión ambiental, los mecanismos se agruparon por afinidad dando como resultado $44 \%$ para proyectos, $31 \%$ para comités y $25 \%$ para otras actividades. El detalle se puede observar en la tabla 11. 
Tabla 11

Respuestas complementarias de la pregunta 5 agrupadas

\section{Clasificación}

Proyectos

Comités

Otras Actividades

Total

Nota. Elaboración propia con base en los resultados.

En la pregunta 27 se contabilizó $75 \%$ (18) de las 24 IEST que mencionaron los programas académicos que consideran más avanzados en la perspectiva ambiental; destacan programas de maestría, cursos y carreras completas, empero, al analizarlas se evidencia que solo $73 \%$ de las respuestas corresponden a programas académicos, $11 \%$ a más bien estrategias institucionales y $17 \%$ a otros como alguna comisión o actividad. Podemos observar el detalle de las respuestas en la tabla 12.

Tabla 12

Agrupación de respuestas complementarias de la pregunta 7

\begin{tabular}{lcc|}
\multicolumn{1}{c}{ Clasificación } & Frecuencia & $\%$ \\
\hline Programa académico & 13 & $72 \%$ \\
\hline Estrategia institucional & 2 & $11 \%$ \\
\hline Otros & 3 & $17 \%$ \\
\hline Total & 18 & $100 \%$ \\
\hline
\end{tabular}

Nota. Elaboración propia con base en los resultados.

\section{Conclusión}

La historia de la educación ambiental (EA) costarricense está muy relacionada al avance en la gestión y/o educación ambiental de las universidades públicas por todas las acciones que desde muchos años atrás se realizaron en búsqueda de la transversalidad de la educación en el medio ambiente.

El impacto de la EA ha sido significativo en términos de población estudiantil (la cual era de $49,1 \%$ del total de la matrícula para el año 2017). La incorporación de las universidades públicas junto con algunos de los centros de estudio superior privados en REDIES ha propiciado una sinergia común en pro de la permeabilización de la sustentabilidad, constatable en los diferentes esfuerzos que se han desarrollado y en el interés y accionar mostrado por sus 20 instituciones adheridas.

Ciertamente, los mismos resultados presentados en este documento evidencian que ha sido positivo para las IEST pertenecer a REDIES, un aspecto clave en la mayoría de los casos para ir desarrollando dentro de cada organización la sustentabilidad.

En la actualidad, REDIES ha logrado conformarse como una alianza de instituciones educativas público-privadas, todas con un deseo común: mejorar con buenas prácticas ambientales de gestión y educación en sus instituciones, además de transferir el conocimiento a la sociedad costarricense. 


\section{Referencias}

Alianza de Redes Iberoamericanas de Universidades por la Sostenibilidad y el Ambiente / Proyecto RISU (2014). PROYECTORISU Definición de indicadores para la evaluación de las políticas de sustentabilidad en universidades Latinoamericanas. https://digi.usac.edu.gt/edigi/digital-02.html

Arguedas, M. (en prensa). La experiencia de la Red Costarricense de Instituciones Educativas Sostenibles (REDIES): mucho más que gestión ambiental. [Manuscrito enviado para publicación]. AMBIENS. Revista Iberoamericana Universitaria en Ambiente, Sociedad y Sustentabilidad. Volumen 3. https://revistas.udca.edu.co/index.php/ambiens/index

Programa Estado Nación (2017). Sexto Informe Estado de la Educación. https://estadonacion.or.cr/informe $/$ ? id=17074dc7-a4c3-40d4-8690-80186 $\underline{8 d 90534}$

Rivero, D. S. (2008). Metodología de la Investigación. Colombia: Editorial Shalom.

Rueda, N. L. (2013). Diagnóstico de la Situación Ambiental de las Universidades Costarricenses. Proyecto de Graduación, Guácimo, Costa Rica: Universidad EARTH.

Sáenz Zapata, O., Plata Rangel, Á., Holguín Aguirre, M., Mora Penagos, W., \& Blanco Portela, N. (2017). Institucionalización del compromiso ambiental de las universidades colombianas. Civilizar: Ciencias Sociales Y Humanas, 17(33), 189-208. https://doi.org/10.22518/16578953.908

Sampieri, R. H. (2010). Metodología de la Investigación. México: McGRAW-HILL. 